\title{
A UK training programme for nurse practitioner flexible sigmoidoscopy and a prospective evaluation of the practice of the first UK trained nurse flexible sigmoidoscopist
}

G S Duthie, P J Drew, M A P Hughes, R Farouk, R Hodson, K R Wedgwood, J R T Monson

\begin{abstract}
Background-Flexible sigmoidoscopy is a technical skill that has been successfully performed by suitably trained colorectal nurse practitioners in the USA. However, no recognised training course exists for nurse practitioners in the UK.

Aims-To design and evaluate a training programme for nurse endoscopists.

Methods-A multidisciplinary committee of nurses and clinicians developed a structured programme of study and practice. This involved a staged process of observations, withdrawals, and ultimately, full procedures. Once training had been completed the nurse practitioner was permitted to practice independently. Patients with colorectal symptoms referred for flexible sigmoidoscopy were examined for the final stages of training and independent practice. A prospective evaluation of the training and practice of the first trained nurse flexible sigmoidoscopist was performed. Barium enema, video, clinical follow up, and histology were used to validate the results of the flexible sigmoidoscopies.
\end{abstract}

Results-The training programme required that 35 observations, 35 withdrawals, and 35 supervised full procedures were performed prior to the development of independent practice. Subsequent to the completion of this programme 215 patients have been examined independently by the nurse practitioner. Ninety three per cent of the examinations were judged successful and pathology was identified in $51 \%$. The nurse endoscopist successfully identified all "significant" pathology whereas barium enema failed to identify pathology in $\mathbf{1 2 . 5 \%}$. There were no complications.

Conclusion-With suitable training nurse endosocopists are able to perform flexible sigmoidoscopy safely and effectively. (Gut 1998;43:711-714)

Keywords: nurse endoscopists; flexible sigmoidoscopy

Correspondence to: Mr G S Duthie, Senior Lecturer/Honorary Consultant Surgeon,

University of Hull Academic

Surgical Unit, Castle Hill

Hospital, Hull HU16 5JQ, UK.

Accepted for publication 13 May 1998
There is an increasing demand for diagn flexible sigmoidoscopy and the potential introduction of flexible sigmoidoscopy screening for colorectal cancer ${ }^{12}$ would probably overwhelm the currently available service. If adequate resources were allocated in terms of equipment there would still be insufficient medically qualified flexible sigmoidoscopists to perform the examinations. One potential solution to this problem is the development of colorectal nurse practitioners trained in flexible sigmoidoscopy. In collaboration with the professional nursing bodies a nationally recognised training scheme has been developed, designed specifically for nurse practitioners wishing to develop the skills necessary to perform flexible sigmoidoscopy. ${ }^{3}$ The structure of the training programme and the prospective evaluation of the practice of the first UK English Nursing Board recognised nurse practitioner flexible sigmoidoscopist are presented in this paper.

\section{Methods}

Symptomatic colorectal patients referred for flexible sigmoidoscopies were recruited for the study. The patients were informed that a nurse would be performing the endoscopy and were given the opportunity to decline entry into the study and have a doctor perform the investigation without delay. A consultant endoscopist was appointed as clinical supervisor and strict protocols concerning the nurse's scope of practice were agreed with the employing Trust who provided the necessary indemnity cover. The local ethics committee granted ethical approval for the study. During the training period and following the transition into independent practice the nurse practitioner and the clinical supervisor collected the relevant data.

\section{THE TRAINING PROGRAMME}

In addition to the practical aspects of flexible sigmoidoscopy a course of study days covering a broad spectrum of theoretical, moral, and legal issues relating to colorectal disease and flexible sigmoidoscopy was included in the training programme.

A standard $60 \mathrm{~cm}$ flexible sigmoidoscope was used throughout the practical training, which was divided into four stages.

\section{Stage I}

This involved the observation and recording of the findings of 35 flexible sigmoidoscopic examinations. The trainee was taught to recognise both normal anatomy and common pathology as well as common endoscopic landmarks. Supervised and unsupervised practice using "colon" models (Key Med Ltd, Essex, 
Table 1 Results of flexible sigmoidoscopy $(n=199)$

\begin{tabular}{ll}
\hline Pathology identified & Number \\
\hline Normal & 97 \\
Diverticular disease & 52 \\
Metaplastic polyps & 32 \\
Inflammatory bowel disease & 8 \\
Stricture & 6 \\
Cancer & 4 \\
Villous adenoma & 1 \\
\hline
\end{tabular}

${ }^{\star}$ Found in one patient with diverticular disease.

UK) was utilised during this period in order to familiarise the trainee with the controls of the endoscope.

Stage II

Once competence on the colon models had been demonstrated and the required number of examinations had been observed the nurse was taught how to withdraw the sigmoidoscope while maintaining visualisation of the bowel wall.

\section{Stage III}

Following the successful completion of 35 supervised withdrawals the trainee performed 35 full examinations including digital examination under direct supervision. At this stage the nurse's endoscopic technique, and knowledge of colorectal anatomy and pathology were assessed. In addition, procedure time and depth of insertion were recorded by the supervising clinician.

\section{Stage IV}

Independent practice was permitted only when the clinical supervisor was satisfied with the competence of the trainee and all previous practical and theoretical stages had been completed satisfactorily. At this stage the trainee was judged to be able to identify both normal and abnormal anatomy and complete the examination within 20 minutes without assistance. The nurse's independently performed examinations were monitored with either a barium enema, reported by a consultant radiologist and performed at the same visit to our "one stop" diagnostic service, video recordings reviewed with the clinical supervisor, and/or clinical follow up. During practical training the nurse was required to maintain a prospective record of practice and participate in regular one to one review of video recorded endoscopies with the clinical supervisor. Case

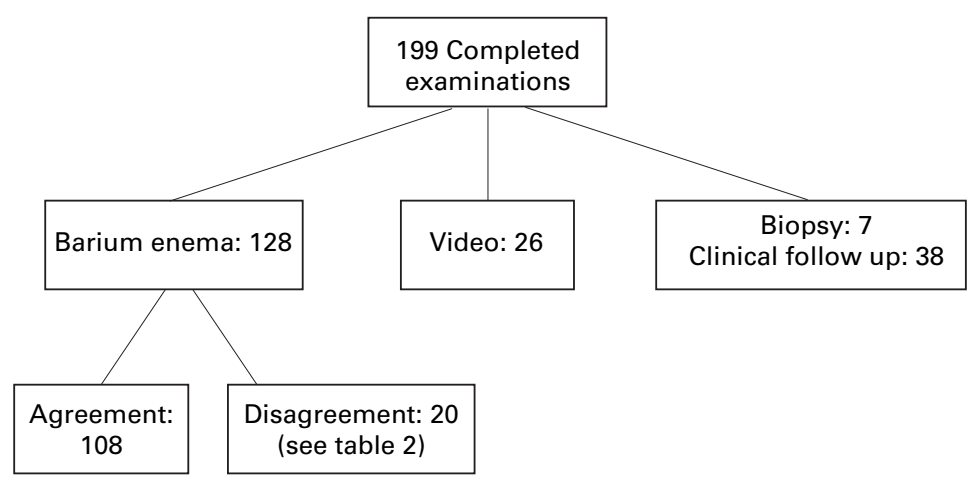

Figure 1 Validation of nurse flexible sigmoidoscopy results.
Table 2 Disagreement between barium enema and flexible sigmoidoscopy

\begin{tabular}{ll}
\hline Flexible sigmoidoscopy & 3 Normal \\
Barium enema & 3 Diverticular disease \\
Flexible sigmoidoscopy & 1 Diverticular disease \\
Barium enema & 1 Normal \\
Flexible sigmoidoscopy & 14 Metaplastic polyp \\
Barium enema & 10 Normal, 4 diverticular disease \\
Flexible sigmoidoscopy & 1 Cancer \\
Barium enema & 1 Diverticular disease \\
Flexible sigmoidoscopy & 1 Villous adenoma \\
Barium enema & 1 Diverticular disease \\
\hline
\end{tabular}

discussions and presentations to other medical and nursing staff were also included. In this preliminary series, in line with our department's policy, sedation was not used, although a sedation module has subsequently been included in the course.

\section{Results}

During the observation phase the nurse performed approximately 10 hours supervised and 20 hours unsupervised practice on the colon models over a three week period. Competency was reached within the required number of examinations at each of the first three stages. A total of 215 patients have now been examined as part of the independent practice of the first nurse endoscopist to complete the course. One hundred and ninety nine examinations $(93 \%)$ were considered successful as classified by depth of insertion or accurate diagnosis following validation. Sixteen $(7 \%)$ were abandoned due to poor bowel preparation $(n=12)$ or pain $(n=4)$. Subsequent endoscopic or radiological review revealed 10 normal examinations, four cases of diverticular disease, and two adenocarcinomas in this group.

Of the 199 successful examinations, 97 (49\%) were normal and pathology was identified in $102(51 \%$ ) (table 1). In 45 patients $(23 \%)$ flexible sigmoidoscopy was the only examination. These patients either underwent a cold biopsy performed by the nurse or have been followed up clinically for a minimum of one year following a normal examination (fig $1)$.

In 20 patients (13\%) there was a disparity between flexible sigmoidoscopy and barium enema results (table 2). In 17 of these patients the flexible sigmoidoscopy was considered diagnostically superior. In the remaining three patients the barium enema identified moderate diverticular disease not recognised by the nurse endoscopist. However, no significant pathology was left undiagnosed by the nurse endoscopist.

During independent practice the sigmoidoscope was passed to a mean insertion depth of $55.5 \mathrm{~cm}$ with a mean examination (withdrawal) time of 6.8 minutes. There were no complications and no patient declined examination by a nurse.

\section{Discussion}

There is little doubt that endoscopy services in the UK are under increasing strain with overbooked inpatient lists and long waiting times for outpatient examinations. Flexible sigmoidoscopy is particularly in demand and with 
the potential introduction of flexible sigmoidoscopy screening for colorectal cancer, ${ }^{12}$ the already overstretched service would be considerably strained. Even if adequate endoscopy facilities were made available there are insufficient medically qualified endoscopists to absorb the increasing workload. The requirements of the "new deal" to reduce doctors' hours ${ }^{4}$ and the Calman report's recommendations to shorten specialist training ${ }^{5}$ will further reduce the availability of juniors to perform service tasks. However, flexible sigmoidoscopy is a technical skill and suitably motivated nonmedical staff, who are able to fulfil the necessary academic and technical requirements, should be able to learn to this technique. In the UK the creation and subsequent expansion of the nurse practitioner grade $^{67}$ represents an opportunity to develop an endoscopically underutilised human resource. In the USA, where nursing has traditionally been more interventional, there are already several centres utilising nurse endoscopy. ${ }^{8-10}$ However, the nursing profession in the UK has only relatively recently established the framework necessary for the further development of nurses into areas traditionally dominated by medical personnel. ${ }^{67}$

The British Society of Gastroenterology supports the development of nurse endoscopy with the provision that appropriate training is available. ${ }^{11}$ The previously available courses in endoscopy were unacceptable for nurse practitioners for several reasons. The different educational background of nurses as opposed to clinicians meant that the theoretical, moral, and legal information contained in a nurse endoscopy course was obviously different to that required in a medical course. In addition to matters relating to flexible sigmoidoscopy nurses must also be taught the theories and issues which underpin and influence the role of the nurse practitioner. ${ }^{12}$ Medicolegally the nurse's practice will be judged to the standard of an experienced endoscopist regardless of medical or nursing background and the breadth and scope of the training programme must be designed to reflect this. Furthermore, the professional bodies responsible for the recognition of nurse training courses are required to ensure that every training course provides a consistent and comprehensive level of training and there are strict guidelines that must be followed before a course can be approved. ${ }^{13}$

The available evidence suggests that the majority of medical trainees become competent after approximately 30-35 flexible sigmoidoscopies, ${ }^{14}{ }^{15}$ and the level of endoscopy episodes included in the training programme reflected this. The nurse assessed in this study achieved competency within the required number of episodes at every stage of training and has not misdiagnosed any serious pathology in 215 independently performed examinations. Validation highlighted the limitations of barium enema alone as a tool for colonic investigation $^{16}$ and would support the recommendation by the Association of Coloproctology ${ }^{17}$ that barium enema and flexible sigmoidoscopy should be combined as the minimum standard for the investigation of the colon and rectum.

While it may be possible to train technicians to perform flexible sigmoidoscopy it was considered more appropriate to utilise the skill base that exists within the UK nursing profession. There is little evidence to suggest that this resource represents only a few highly motivated individuals. Eighteen nurses have completed the three courses we have already organised and our future courses are currently overbooked. While our course is now not the only recognised flexible sigmoidoscopy course for nurses, it was the first English Nursing Board recognised course. As such, we have had many enquiries from other units wishing to develop their own ENB recognised courses.

It must be emphasised that these nurses are not regarded as technicians and are encouraged to develop their practice in other areas. This is essential if colorectal nurse practitioners are to maintain interest and enthusiasm in their role and are not to be perceived as moving from "handmaiden" to "technical functionary". ${ }^{18}$ Indeed, the degree of autonomy practised by the trained colorectal nurse practitioners is in line with the proposed future of nursing and represents a true shift in the care paradigm from "multidisciplinary" to "interdisciplinary". As such, in many centres the nurses we have trained in flexible sigmoidoscopy are employed as colorectal nurse practitioners with skills in counselling, patient education, prevention, and other related areas.

In line with our department policy the nurse practitioner did not use sedation in order to perform the flexible sigmoidoscopies included in this series. However, following the introduction of our upper gastrointestinal course, a separate sedation module has now been developed. Having completed the module, our current upper and lower gastrointestinal nurse practitioners are now able to administer midazolam independently. No doctor is required either to prescribe or administer this sedation which is given by the nurse according to a strict protocol.

There is little apparent saving in terms of salary when comparing a nurse practitioner with a junior doctor. However, the nurse can be utilised in other areas of colorectal practice and will not be changing post and require retraining every 6-12 months.

The training and introduction of nurse endoscopists was initially greeted with caution by the junior medical staff who were concerned that their training may be compromised. However, the provision of a dedicated nurse endocopy list led to a subsequent reduction in pressure of time on the teaching lists for the junior medical staff and also released a senior member of staff to teach. In fact, it was not uncommon for inexperienced junior medical endoscopists to attend the nurse endoscopy lists in order to observe the nurse's practice prior to beginning formal training with the consultant endoscopist. Indeed, the junior medical staff have subsequently requested full integration into certain modules of the course. 
In conclusion, we have shown that by utilising a comprehensive training programme, nurse practitioner flexible sigmoidoscopy can be successfully carried out on an independent basis. As such colorectal nurse practitioners may soon be able to provide a comprehensive flexible sigmoidoscopy service as part of their contribution to the "interdisciplinary" care of patients with colorectal disorders.

M A P Hughes is a nurse endoscopist/counsellor supported by East Yorkshire Hospitals NHS Trust. P J Drew was supported by a Northern and Yorkshire Regional Healthy Authority Research Fellowship. This work was presented at the Surgical Research Society, January 1996, and Association of Surgeons of Great Britain and Ireland, May 1996.

1 Austoker I. Screening for colorectal cancer. BMf 1994;309:382-6.

2 Atkin WS, Cuzick I, Northover JMA, et al. Prevention of colorectal cancer by once-only sigmoidoscopy. Lancet 1993;341:736-40.

3 Duthie GS, Hughes MAP. Colorectal screening course. ENB 9N81 (ISBN 13986122803 ).

4 NHS Management Executive. Funior doctors. The new deal. London: NIHS Management Executive, 1991.

5 Working Group on Specialist Medical Training (K Calman, chairman). Hospital doctors: training for the future. London: Department of Health, 1993.

6 United Kingdom Central Council for Nursing, Midwifery and Health Visiting. The scope of professional practice. London: UKCC, 1992.
7 Department of Health and Social Security. The extending role of the clinical nurse. Legal implications and training requirements. London: DHSS, 1977 (HC(77)22)

8 Maule WF. Screening for colorectal cancer by nurse endoscopists. N Engl f Med 1994;330:183-7.

9 Rosevelt J, Frankl H. Colorectal cancer screening by nurse practitioners using $60 \mathrm{~cm}$ flexible sigmoidoscope. Dig Dis Sci 1984;29:161-33.

10 Schroy PC, Wiggins TW, Winawer SI, et al. Video endoscopy by nurse practitioners: a model for colorectal screening. Gastrointest Endosc 1988;34:390-4.

11 Report of the British Society of Gastroenterology Working Party. The nurse endoscopist. British Society of Gastroenterology, 1994

12 Hughes MAP. Key issues in the introduction of nurse endoscopy. Nursing Times 1996;92:38-9.

13 United Kingdom Central Council for Nursing, Midwifery and Health Visiting. The council's standards for education and practice following registration. Programmes of education leading to qualification of specialist practitioners. London: UKCC, to qualifica

14 Cass OW, Freeman IL, Peline CI, et al. Objective evaluation of endoscopy skills during training. Ann Intern Med 1993;113:40-4.

15 Hawes R, Leliman GA, Hast J, et al. Training resident physicians in fibreoptic sigmoidoscopy. How many supervised examinations are required to achieve competence? $A m \mathcal{7}$ Med 1986;30:465-70.

16 Brewster NT, Grieve DC, Saunders JH. Double contrast barium enema and flexible sigmoidoscopy for routine colonic investigation. Br F Surg 1994;31:445-7.

17 Association of Coloproctologists. Guidelines for the management of colorectal cancer. Association of Coloproctologists, June 1996.

18 Denner S. Extending professional practice: benefits and pitfalls. Nursing Times 1995;91:27-9. 